\title{
e-Phaïstos
}

e-Phaïstos

Revue d'histoire des techniques / Journal of the history

of technology

III-1 | 2014

Varia

\section{Le patrimoine industriel pétrolier vénézuélien : un patrimoine toujours en activité}

Venezuelian Oil Industrial Heritage: a heritage still operating

\section{Lucía Sánchez Figueroa}

\section{(2) OpenEdition}

Journals

Édition électronique

URL : http://journals.openedition.org/ephaistos/708

DOI : 10.4000/ephaistos.708

ISSN : 2552-0741

Éditeur

IHMC - Institut d'histoire moderne et contemporaine (UMR 8066)

Édition imprimée

Date de publication : 1 juin 2014

Pagination : 112-122

ISSN : 2262-7340

Référence électronique

Lucía Sánchez Figueroa, «Le patrimoine industriel pétrolier vénézuélien : un patrimoine toujours en activité », e-Phaïstos [En ligne], III-1 | 2014, mis en ligne le 24 novembre 2016, consulté le 19 avril 2019. URL : http://journals.openedition.org/ephaistos/708 ; DOI : 10.4000/ephaistos.708

Tous droits réservés 


\title{
Le patrimoine industriel pétrolier vénézuélien : un patrimoine toujours en activité
}

\author{
Lucía Sánchez Figueroa \\ PDVSA Ingeniería y Construcción \\ Vénézuela
}

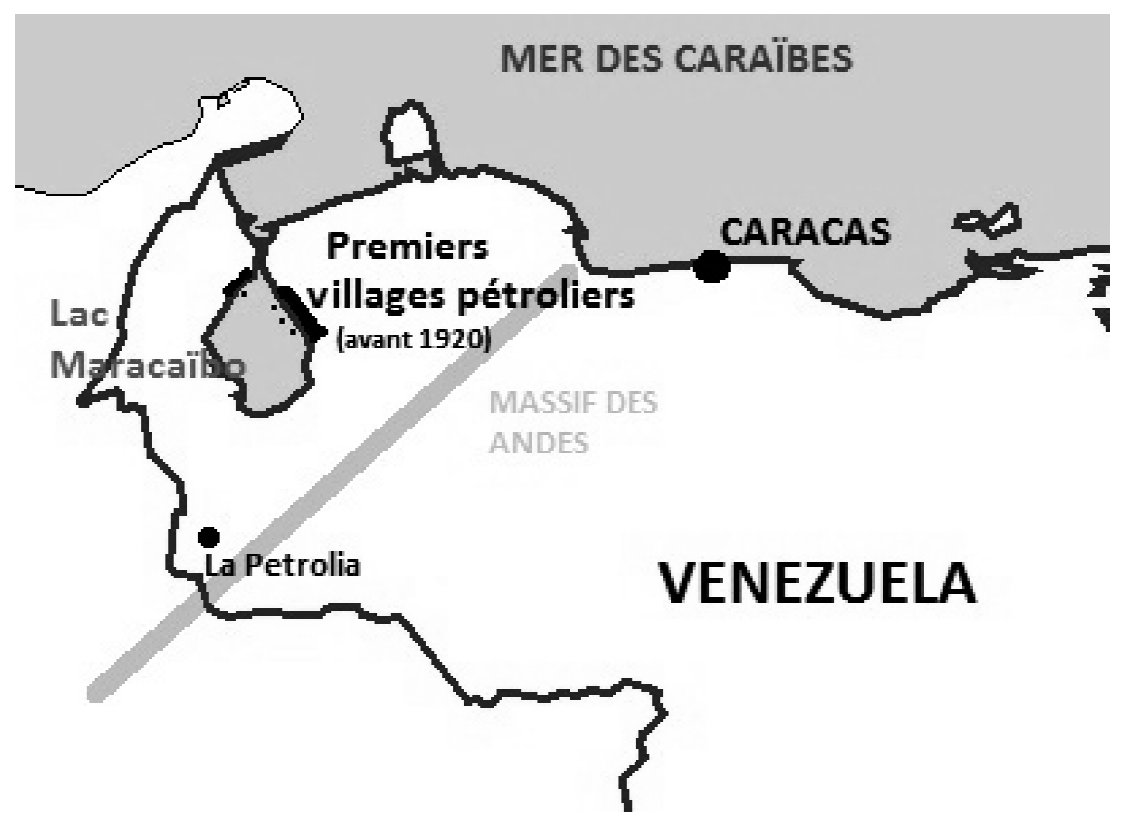

Figure 1 : Carte de localisation (Nord-Ouest du Venezuela) pétrole/ électricité/ alliages. De façon générale, l'énergie a représenté pour les sociétés de tous temps, un instrument indispensable pour moduler les éléments de sa subsistance. C'est elle, avec son développement technique, qui détermine et garantit les modèles de civilisation. Le progrès ou le recul d'une nation, ses machines de défense et la qualité de vie de la population, sont intrinsèquement liés au profit de ses ressources. C'est sa potentialité et sa disponibilité qui conditionnent son degré d'utilisation.

Le pétrole, énergie et carburant principal de la seconde industrialisation, a permis les échanges entre les nations puissantes à un niveau mondial. Mais l'activité pétrolière ne constituait pas seulement un échange économique. Elle a représenté un changement abrupt de complexe

Nous ne saurions aborder le sujet du patrimoine industriel pétrolier vénézuélien sans replacer le pétrole au sein de l'histoire des techniques. Le pétrole apparaît, à partir de 1860 , comme un élément central du système technique ${ }^{1}$ de la seconde industrialisation, c'est-à-dire, le système technique, social et territorial. L'expérience des champs pétrolifères a reconfiguré les identités régionales, et a introduit une nouvelle façon de vivre (reconfiguration des espaces publics et privés, changement de la notion du temps, etc.). 
Aujourd'hui, après 120 années d'exploitation de son pétrole et 100 ans après le début de la Première Guerre Mondiale, le Venezuela possède encore la réserve de pétrole la plus importante du monde. L'industrie pétrolière a créé (et créé encore) des espaces de production, qui, jusqu'à aujourd'hui, constituent la base de l'économie du Venezuela. Pourtant, si dans l'imaginaire collectif, il n'existe pas d'autre regard que le regard économique sur cette industrie, les espaces anciens et reconfigurés, constituent de fait un patrimoine industriel à mettre en valeur.

\section{La Petrolia, première compagnie pétrolière vénézuélienne}

De la naissance de la République à la fin du XIXe siècle, le café et le cacao ont représenté pour le Venezuela les principaux produits d'exportation.
Pendant cette période, l'économie vénézuélienne était soumise à leurs fluctuations de prix sur les marchés internationaux ; et ce jusqu'en 1914, date à laquelle la Caribbean Petroleum Company (CPC, groupe Shell) a démarré l'exploitation intensive du pétrole. Cependant, il existait déjà une entreprise entièrement vénézuélienne, La Petrolia, qui exploitait le pétrole quand les concessionnaires sont arrivés, mais il demeurait impossible pour cette compagnie d'entrer en compétition avec les entreprises étrangères et leurs technologies conçues pour l'exploitation de masse.

Cela dit, fondée en $1878^{2}$ par Manuel Pulido, José Baldó, Ramón Maldonado, Carlos González, José Villafañe et Pedro Rincones, La Petrolia a réussi à mettre en place toutes les opérations de la chaîne opératoire du pétrole de son extraction à son raffinage. $\mathrm{Si}$, dans les premiers temps, seul le pétrole affleurant était récupéré, en utilisant des
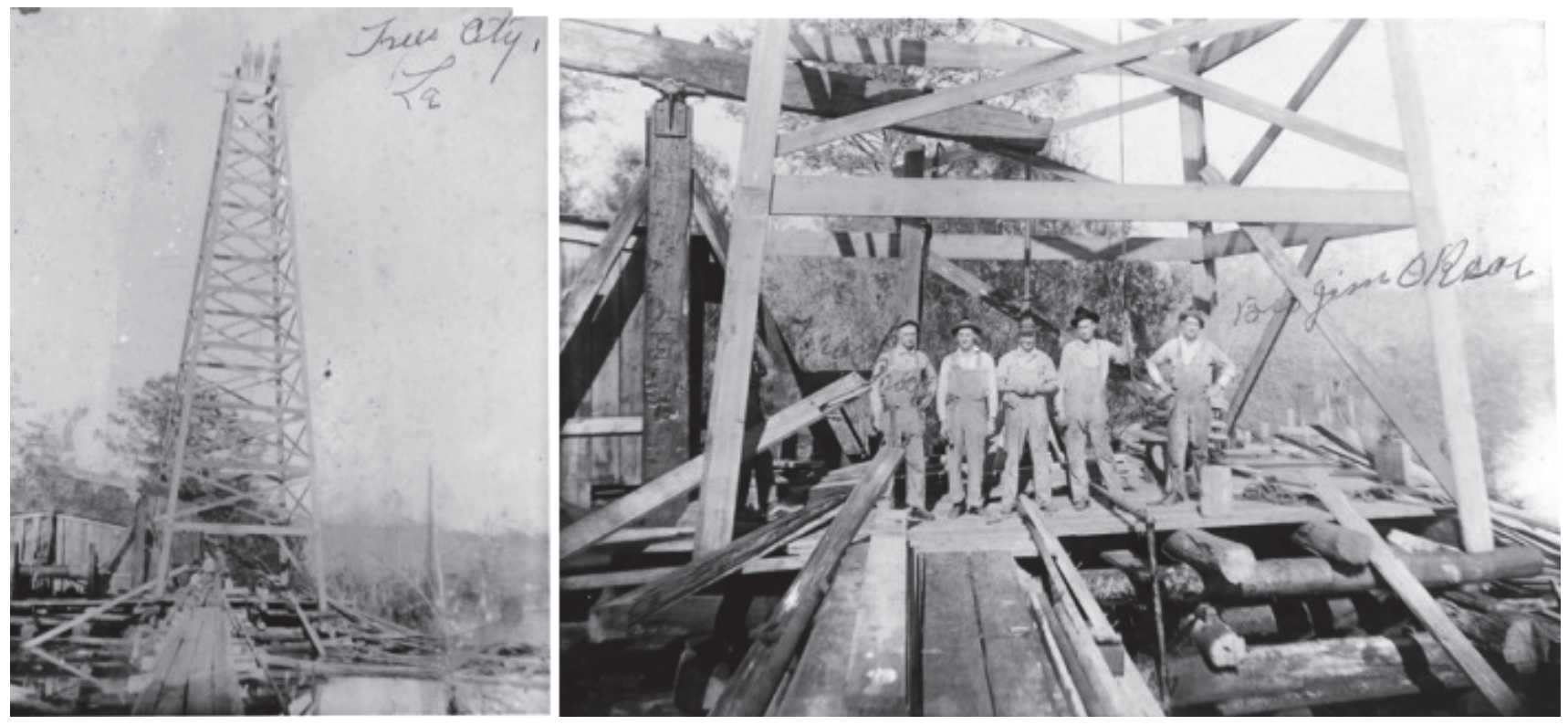

Fig. 2 : L'Eureka de La Petrolia du Tachira. Source : Arnold, R.; Macready, G. A. y Barrington, T. W., The First Big Oil Hunt, Venezuela 1911-1916, Vantage Press Inc., New York, 1960. 
couvertures selon une technique indigène, dès 1883 , l'entreprise avait achevé son premier puit, l'Eureka (6om de profondeur), en utilisant des outils importés, et raffinait du pétrole pour fabriquer du kérosène. Dès 1884, La Petrolia produisait ainsi 6 barils par jours.

Suite au coup d'état du général Juan Vincente Gómez en 1908, favorable aux britanniques et aux américains, l'exploitation du pétrole vénézuélien fut ouverte aux entreprises étrangères. En 1917, la Caribbean Petroleum Company a installé une raffinerie près de la localité de San Lorenzo dans la province du Zulia, à l'ouest de la région où $L a$ Petrolia avait déjà le monopole du raffinage, de l'approvisionnement et de la distribution des combustibles du pétrole depuis 30 ans. Ainsi, pendant que La Petrolia poursuit ses activités, d'autres compagnies arrivent sur le territoire vénézuélien. Puis, en 1922, un évènement confirma l'extraordinaire potentiel minier du Venezuela : l'éclatement du puits Barroso 2 par la Venezuelan Oil Concessions dans le champ La Rosa de la province du Zulia. Cet évènement produisit 100.000 barils par jour pendant 9 jours.

Sept ans plus tard, en 1929, il y avait 73 compagnies qui cherchaient de façon isolée du pétrole. Par contre, seules celles qui font aujourd'hui partie du groupe Shell ont réussi dans leur entreprise. De plus, la production annuelle de pétrole a augmentée pendant ces années pour passer de 2.200.000 barils à 137.000.000 de barils. À cette époque, le Venezuela était devenu le plus grand exportateur de pétrole du monde et le deuxième plus grand producteur de pétrole, après les États-Unis. Le volume des exportations de pétrole est passé de 1,9\% à 91,2\% du pétrole produit entre 1920 et 1935.

Cette capacité d'exploitation massive des entreprises étrangères a marqué la fin de $L a$ Petrolia. En effet, si l'entreprise a su mécaniser la production, c'est d'abord grâce à l'importation d'outils américains. Or, une fois les outils cassés, la plupart étaient abandonnés et si quelques-uns étaient adaptés, aucuns n'étaient réparés. Ainsi, ils ont conçus des outils très spécifiques à leur zone de monopole, et les infrastructures mises en place furent vite insuffisantes au regard de la demande accrue et aux rendements des nouvelles techniques utilisées par les concurrents. En 50 ans de production, nous n'observons donc ni appropriation ni hybridation des techniques. Les raisons de ce retard fut l'incapacité à innover pour créer une filière technique adaptée, due, entre autres choses, à la mort des fondateurs et à des héritiers moins intéressés par les innovations techniques et un moindre appui du gouvernement. Ainsi, en 1934, le gouvernement n'a pas renouvelé sa concession à l'entreprise et la concession pour opérer les terrains de La Petrolia fut attribuée à la Royal Dutch Shell en 1938.

\section{L'ère du pétrole}

En 1914, avec la Première Guerre Mondiale, s'initie la mécanisation du champ de bataille avec une demande conséquente de pétrole pour alimenter les machines de guerre. Par contre, pour le Venezuela, ce développement de l'industrie a signifié un recul. De 1914 à 1917, quelques gisements de pétrole ont été découverts sur l'ensemble du pays, mais à cause de l'effort de guerre, et de l'achat d'outils et de machines, quelques compagnies pétrolières se sont vues obligées de renoncer à la perforation, et ce même après la guerre.

En 1914 toujours, la Caribbean Petroleum Company a annoncé la découverte du puits Zumaque 1. Il était dans les alentours de San Lorenzo dans la province du Zulia et il est arrivé à produire une totalité de 500570202 barils de pétrole. À partir de ce moment, le Venezuela est devenu la cible des investissements internationaux, tant pour ses considérables réserves de pétrole brut, que pour l'instrumentalisation de la "politique pétrolière la plus libérale de toute l'Amérique latine »3. À cette politique libérale fut adjointe une 


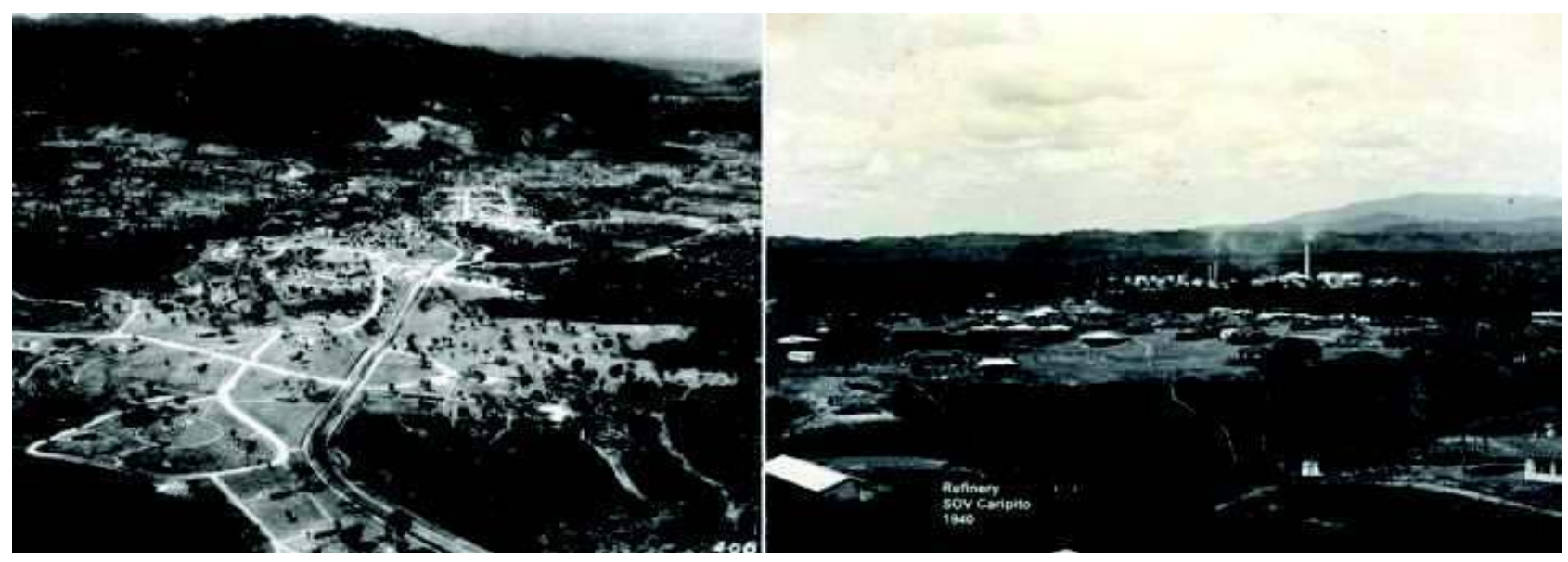

Figure 3 : Village pétrolier Caripito, 1935. Source : Valery, 1980 (cf. bibliographie)

politique d'aménagement: à partir de 1917, les recettes fiscales pétrolières, comme l'explique la Revue Technique du ministère des travaux publics, ont été destinées à la construction des « voies pétrolières ", visant à faciliter l'accès des secteurs vierges du territoire où il $\mathrm{y}$ avait du pétrole. Cet effort de consolidation des voies de communication était indispensable pour l'exploration, le forage et la production pétrolière sur la côte orientale du lac Maracaibo, très riche en pétrole.

Ces nouveaux ponts, ports, aéroports, terminaux de chemins de fer, et oléoducs permirent aux entreprises pétrolières concessionnaires étrangères d'établir des villages pétroliers, où les bureaux et les résidences du personnel de direction étaient séparés par un mur des lieux de résidence des ouvriers et de leurs familles.

Les différences entre le secteur du personnel technique et le secteur du personnel ouvrier étaient très marquées. À partir de 1928 , le président du Venezuela, Juan V. Gómez, commence à recevoir de multiples plaintes concernant les inégalités profondes de conditions de vie entre les travailleurs étrangers (cadres) et nationaux (ouvriers) au sein des villages pétroliers. C'est pourquoi, tandis que l'activité pétrolière se stabilisait, les installations ont été mises en conformité, selon la vision du monde "moderne ». Ainsi ont été construites des demeures certes peu salubres, mais qui sont devenues de petits centres offrant les services d'une ville, parfois gratuits et subventionnés par l'entreprise. Ces nouveaux «villages » étaient constitués de résidences ouvrières, de résidences pour les techniciens et les célibataires, avec les services éducatifs, religieux et récréatifs correspondants, avec des bureaux, des ateliers, et des réservoirs et avec des installations auxiliaires : magasins, restaurants et clubs, etc. Le modèle était celui du paternalisme européen du XIX ${ }^{\mathrm{e}}$ siècle, permettant de faire accepter aux ouvriers leur condition laborieuse, tout en se préservant de mouvements sociaux. En effet, du point de vue humain, idéologique et territorial, les entreprises promouvaient la moralité, l'individualisme, la religion et l'obéissance au patron dans un environnement quadrillé, dans lequel il était plus facile de contrôler toute activité hors norme, comme les révoltes syndicales, que dans une ville. 


\section{Les villages pétroliers}

Les villages pétroliers constituent un élément clé dans l'histoire du pétrole au Venezuela et un des aspects le plus critiqué à partir de 1920. "Depuis le début, ils étaient des noyaux résidentiels isolés où la clôture était, en même temps, le symbole et la barrière qui séparait les différentes zones du village et aussi le village de l'extérieur $\gg 4$ : il y avait des espaces séparés selon la catégorie du travailleur (directeurs, techniciens et ouvriers) et en plus, il existait une importante dichotomie avec ce qui se passait à l'extérieur. Au final, les villages pétroliers ont généré deux Venezuela. Le premier, le Venezuela à l'extérieur de la clôture, était composé d'espaces ruraux, avec une agriculture en décadence, avec une architecture coloniale ou issue des différentes ethnies, où se côtoyaient des classes sociales très différentes. Le deuxième, le Venezuela à l'intérieur de la clôture, était celui de l'abondance, des services, des revenus supérieurs, et dans lequel la disparité sociale était très bien gérée pour ceux qui observaient de l'extérieur. Pour ces derniers c'était le «Venezuela de la modernité». Cette situation a généré dans tout le pays, une grande migration de la population, surtout des régions rurales, qui voulaient faire partie de cette illusion réalité de l'intérieur de la clôture.

Le concept de village pétrolier (voir fig. 3), en tant que communauté fermée, est resté inchangé jusqu'en 1937, où par décret présidentiel, la construction d'un nouveau modèle de ville a été exigée. De plus, du fait qu'à partir de 1938 (nationalisation du pétrole mexicain), le Venezuela

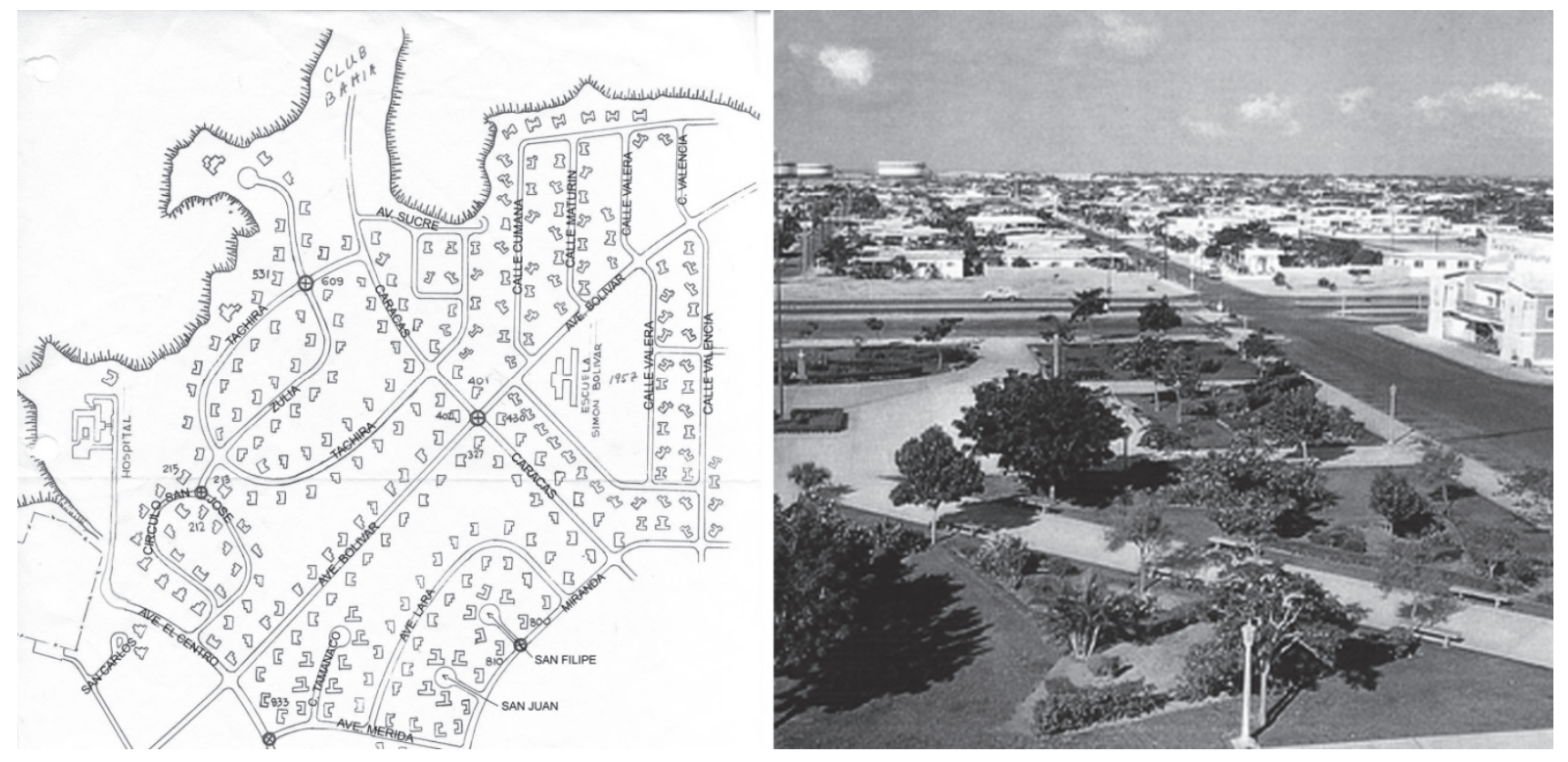

Figure 4 : La planification de la ville, Judibana, projetée par l'atelier Skidmore, Owings et Merril. Source : Valery, 1980 (cf. bibliographie) 
demeurait le seul pays d'Amérique latine autorisant l'exploitation pétrolière par des entreprises étrangères, ces dernières se devaient d'améliorer les conditions de vie des travailleurs pour assurer la continuité de leurs affaires au Venezuela.

Or, depuis le début du XXe siècle, les idées visant à réconcilier les avantages de la campagne et de la ville, visant à améliorer les conditions de logements ouvriers des villes industrielles, et visant à prendre en compte le besoin d'hygiène et de salubrité, sont largement discutées. Ces idées sont bien ancrées dans l'urbanisme de l'époque, héritier des travaux d'E. Howard'5. Grâce aux adeptes de Howard et du "Mouvement des Cités-jardins » se sont propagés les bénéfices de l'urbanisation résidentielle de faible densité, où le travail et le logement continuaient à être liés. Ce modèle a été très bien accueilli par les entrepreneurs de l'époque, ainsi que par les entreprises transnationales au Venezuela.

Peu de villes industrielles ont été construites suivant ce schéma de cité-jardin, à cause de la maintenance qu'elles nécessitaient, mais certaines expériences se sont révélées convaincantes. Ainsi sont apparues des villes ouvertes, plus ou moins intégrées, comme la ville d'Ojeda en 1939 et d'autres cités-jardins d'échelle mineure. La ville d'Ojeda est devenue un des ports les plus importants du pays grâce à l'activité pétrolière. Après sa construction, elle a été reconnue comme étant un effort pionnier dans la planification urbaine au Venezuela. Par ailleurs, l'exécution de projets comme la ville d'Ojeda, a fait place à d'autres villes ouvertes planifiées, plus petites mais à l'impact important, qui sont apparues comme une partie du Projet Urbain du Centre d'Amélioration Paraguaná, actuellement la plus grande raffinerie du monde. Les villes construites comme une partie du complexe de la raffinerie sont Judibana (1950, ancien Champ Creole) et Cardón (1949, ancien Champ Shell). Ces communautés ouvertes, (voir fig. 4) associées à cette époque uniquement à des développements pétroliers, en matière de trame urbaine, constituaient un dessin nouveau : basses densités, logements isolés entourés de jardins, couloirs de services, grands parcs, un tracé organique, où l'emploi de la courbe contraste avec le quadrillage traditionnel.

L'architecture et l'urbanisme employés étaient très marqués par les paramètres modernes d'un style international (voir fig. 4), avec des matériaux nouveaux comme le béton armé, mais appliqués dans un désert d'une façon originale en contraste avec la manière traditionnelle de construire en terre pisée héritée de la colonisation espagnole. L'introduction du béton armé a bouleversé l'architecture, il est encore aujourd'hui le matériau le plus utilisé et a contribué à ce que les constructions en pisée fassent partie du passé et soit considérées comme du patrimoine.

\section{Les paysages du pétrole}

Après la fin du XIXe siècle, toutes les nouvelles villes créées au Venezuela, à l'exception de Ciudad Guayana, l'ont été grâce à l'intense activité pétrolière. Bien que les villages industriels aient commencé comme des installations précaires, ils sont devenus la pièce maîtresse du logement social $\mathrm{du} \mathrm{XX}^{\mathrm{e}}$ siècle au Venezuela. Les résultats de ce processus d'urbanisme intense lié au pétrole sont les dizaines d'établissements qui ont radicalement modifié la géographie urbaine du pays.

En 1950, il y avait au lac Maracaibo environ 2000 puits pétroliers dont les tours géométriquement disposées, s'étendaient au-delà de l'horizon ${ }^{6}$, produisant ce qui a été considéré comme une "des vues les plus imposantes du monde industriel $\gg 7$ (voir fig. 5). À partir de cette époque, l'architecture pétrolière est devenue synonyme de légèreté, flexibilité, standardisée. Auparavant, ce type de construction n'était pas connu au Venezuela. 
On peut affirmer qu'entre 1917 et 1960, l'activité pétrolière des concessionnaires a participé de façon directe et puissante au développement de l'industrie vénézuélienne et de ses activités associées: les chemins de pénétration, les gisements, les puits, les

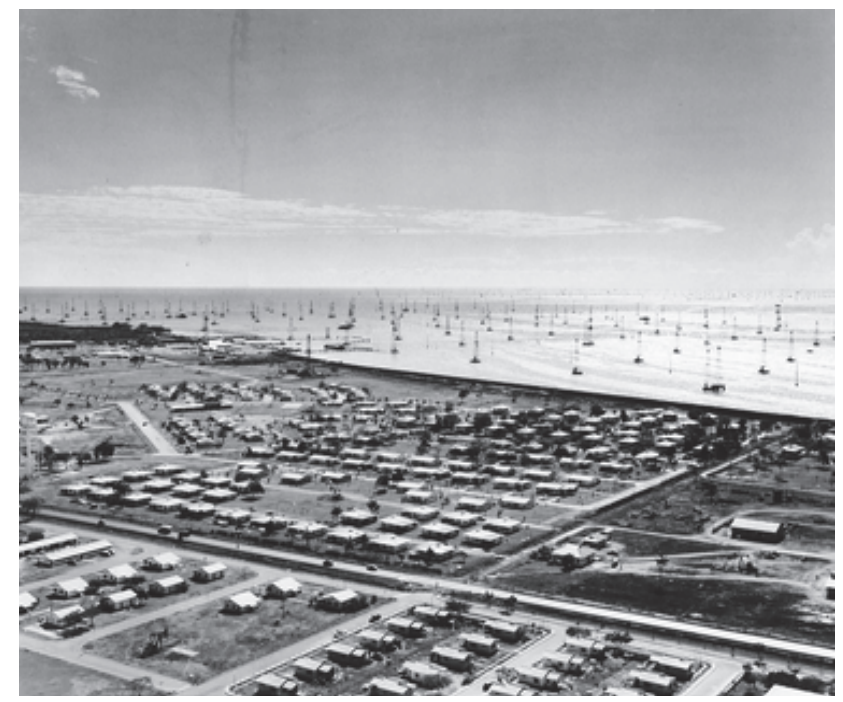

Fig. 5 : Village pétrolier Tia Juana. Source : Valery, 1980 (cf. bibliographie)

gazoducs, les oléoducs, les villages industriels, comme ceux des faubourgs nord-américains. Tous ces éléments ont fait partie d'une nouvelle couche de notre géographie, faisant passer les patios de séchage du café ou du cacao de l'imaginaire industriel à un véritable paysage pittoresque évoquant le passé.

Les années suivantes, les concessionnaires ont commencé une phase connue sous le nom d'« intégration». Les concessionnaires avaient la responsabilité, selon l'article 117 du contrat collectif qui était entré en vigueur à ce moment, d'intégrer les villages pétroliers aux communautés voisines pour qu'ils puissent avoir accès aux services, aux équipements urbains et aux logements. Ce processus, qui a duré 14 ans, a été réalisé en coordination avec les municipalités respectives.

Après la nationalisation de l'industrie pétrolière en 1976, plusieurs villages pétroliers ont été abandonnés ou sont entrés dans un processus progressif de détérioration, car, étant situés à l'intérieur de municipalités très pauvres, ils n'ont pas pu être maintenus état. Donc, ce qui a constitué pour le pays un riche patrimoine qui reflète l'évolution du Venezuela industriel et des échanges multiculturels est aussi une charge administrative et est davantage perçu comme tel. Pourtant, nous ne pouvons pas continuer à voir le patrimoine dans le sens restreint de bâtiments ecclésiastiques datant de l'époque coloniale. Le patrimoine industriel intègre des paysages culturels constitutifs de notre réalité.

\section{Du champ pétrolifère au Patrimoine industriel}

Selon la définition du patrimoine industriel du $\mathrm{TICCIH}^{8}$, celui-ci implique une approche pluridisciplinaire, permettant de comprendre les relations entre l'espace bâti, le savoir-faire technique et le fait social. En revanche, il existe des approches différentes selon les pays. C'est totalement compréhensible parce que bien qu'ils partagent une partie de leur histoire, en ce qui concerne la révolution industrielle. Ils ne sont pas entrés en même temps dans cette phase d'industrialisation, donc, ils ont aussi une histoire indépendante liée à leur territoire, à leurs activités économiques, à leur société, et bien sûr, à leur culture.

En Amérique latine, nous ne nous trouvons pas dans la même temporalité qu'en Europe. Comme nous l'avons mentionné précédemment, le développement technique n'a pas suivi pas le même parcours chronologique. De plus, l'industrialisation n'est pas un résultat lié au développement technique, ancré dans une culture du progrès. Il 
s'agissait d'une industrialisation imposée par les entrepreneurs étrangers, publics et privés, qui voulaient étendre leur pouvoir économique. De ce fait, les exemples de patrimoine industriel sont différents et très divers.

Notons aussi qu'il ne s'agit pas d'un processus homogène et on ne peut pas englober dans un même article toute l'Amérique latine. Il y a des pays qui ont des vestiges riches de l'ère proto industrielle précoloniale ou coloniale, et d'autres qui se caractérisent par un patrimoine proprement industriel développé entre la fin du XIX et le XXe siècle. Plusieurs pays sont en effet passés de structures économiques dépendantes de la colonisation directement à celles liées à l'industrialisation étrangère. L'une des conséquences de cette constitution " exogène » du patrimoine industriel est qu'il suscite, de ce fait, un très fort rejet. Ces derniers décrivent la réalité du patrimoine industriel au Venezuela, pays avec des paysages industriels de grande échelle, et dont l'industrie est encore en activité.

Y a-t-il une réflexion sur le patrimoine industriel en corrélation avec les réalités latino-américaines et prenant en compte leurs différences ? Est-ce qu'il y a une décantation des référents pris de l'expérience européenne? Existe-t-il une étape préalable au diagnostic intégral nécessaire pour les besoins culturels (sociaux et du territoire) ? Sont-ils entrés dans ce cycle de sur-patrimonialisation, où tous devient musée ou site touristique ? Ces questions sont peu posées, et il semble qu'il faille réfléchir davantage sur l'importance de l'adaptation des concepts et des expériences européennes aux contextes différents pour former une conscience du patrimoine industriel qui enrichisse nos échanges et qui implique une inclusion sociale. Sinon, on restera toujours dans l'aspect esthétique du patrimoine. Ce sont ces discussions sur le patrimoine industriel mondial qui poussent désormais à l'analyse des cas comme celui du Venezuela.

\section{Le patrimoine industriel vénézuélien}

L'industrialisation au Venezuela est synonyme de pétrole. C'est le moteur de notre économie mono-productrice depuis 100 ans. La différence principale entre le patrimoine industriel au Venezuela et la majorité des cas et des réflexions faites au niveau mondial sur la question du patrimoine industriel, est le fait que l'industrie qui lui a donné naissance est encore très présente et en activité. De ce fait, les structures ne sont pas abandonnées et il n'existe pas de friche industrielle complète. Cela a l'avantage de permettre de penser une solution, une possibilité, avant le processus de désindustrialisation tel que d'autres pays l'ont connu, afin de limiter l'étape traumatique. Dans ce cadre, la première étape logique devait être celle de l'inventaire et du diagnostic intégral du patrimoine industriel.

Cependant, la notion de patrimoine industriel n'est pas développée au Venezuela. L'étude du patrimoine continue à être celle des constructions ecclésiastiques de la période coloniale ou du patrimoine moderne de style international, bien sûr lié indirectement aux richesses du pétrole. De plus, il n'existe pas de regard exhaustif pour connecter l'ensemble des sites et obtenir une vision macroscopique de l'ensemble du complexe technique. Il manque aussi une connaissance de l'histoire des techniques, nécessaire pour analyser l'histoire pétrolière et le patrimoine qui lui est lié sous plusieurs facettes: économique, sociale, architecturale, technique, etc.

Ainsi, comme il n'existe pas de conscience collective sur le patrimoine industriel et que l'industrie pétrolière est encore en activité, ces paysages culturels, d'une richesse incalculable avec des cartes mentales très fortes, sont perçus seulement comme des espaces de production.

Cependant, le potentiel de développement est présent. Tous ces domaines appartiennent totalement à l'Etat, à l'exception des maisons qui ont été vendues dans le passé à leurs anciens 
propriétaires. Or, si de petits efforts sont réalisés par l'entreprise pétrolière nationale, PDVSA, pour raviver l'intérêt pour le patrimoine industriel, il s'agit toujours d'activités dispersées, comme les expositions sur l'histoire économique ou sociale du pétrole, et quelques visites guidées de raffineries. Il existe bien une intention de faire un musée du pétrole, mais à Caracas, c'est-à-dire en dehors de l'espace industriel, donc totalement décontextualisé, alors qu'au sein de l'espace de travail, des possibilités infinies sont envisageables. De même, l'entreprise compte bien un département $\mathrm{du}$ patrimoine, mais il s'occupe principalement du patrimoine urbain architectural, encadré par le programme des " semailles pétrolières 9 ».

Cet intérêt focalisé sur l'architecture est aussi le cas d'un projet lié au patrimoine industriel: la création d'un centre culturel dans l'ancienne maison du chef de la raffinerie, dans le village industriel de Cardon à Paraguaná. Bien que la reconversion ait respecté plus ou moins l'infrastructure, on note des inconsistances par rapport à une opération liée au patrimoine industriel. Il n'existe aucun espace pour raconter son passé industriel ou pour l'envisager dans une proposition a échelle urbaine, pour la mettre en valeur ou la placer dans un réseau du patrimoine industriel ou au moins l'inventorier.

Ce manque de prévision concernant l'avenir du patrimoine industriel ne traduit pas une absence de volonté de PDVSA, parce qu'avec les différentes activités réalisées, nous constatons que l'intention existe. Le cœur du problème est que, jusqu'à aujourd'hui, il existe qu'une personne dans toute l'entreprise formé pour réaliser ce travail. S'il existe bien des formations en architecture, archéologie, histoire ou ingénierie, la réalité est qu'au Venezuela, il n'existe pas de structure pour former des professionnels sensibilisés au patrimoine industriel. Cette lacune se retrouve dans les universités, à l'Institut du Patrimoine Culturel, au sein de l'entreprise, et dans la communauté. L'approche du patrimoine industriel est liée au patrimoine architectural, et donc limitée à l'analyse du bâti, à l'esthétique, mais sans vision du territoire, du paysage ou du complexe technique.

Actuellement, ceux qui sont en train de faire les démarches pour la valorisation du patrimoine industriel et qui travaillent sur la création du TICCIH Venezuela, ont été formés à l'étranger. L'une d'entre eux travaille à PDVSA. Il y a en tout deux chercheurs dédiés au patrimoine industriel et une dizaine de chercheurs intéressés par ce nouveau sujet au Venezuela. En conséquence, il est nécessaire de renouveler les structures et il est nécessaire de commencer par les universités afin de mettre en valeur notre patrimoine.

Le contenu des études ne correspond plus à la réalité du pays. Ils ont été dessinés sur le modèle proposé par les concessionnaires où les professionnels vénézuéliens étaient prêts pour le travail pratique, mais n'avaient pas besoin d'être formés à la recherche, puisque les concessionnaires apportaient leurs technologies, puis les vendaient après la nationalisation. En conséquence, les universités vénézuéliennes ont une activité de recherche presque inexistante. Ce modèle, transféré à PDVSA, a formé 90 ooo travailleurs directs et contribue à la vision, dans l'imaginaire collectif, du patrimoine industriel comme espace de production, n'étant pas à protéger. Quand fut signée la convention entre l'entreprise et les universités au début du XXI ${ }^{\mathrm{e}}$ pour encourager le développement technique, on vit qu'elles n'étaient pas préparées pour affronter ce défi.

Aujourd'hui l'entreprise affirme la souveraineté pétrolière, puisque il y a un contrôle majeur des activités, mais elle oublie qu'au niveau de la technologie et de l'innovation, le pays dépend encore des entreprises étrangères. L'institut de recherche de PDVSA, l'Intevep, a développé plusieurs innovations de grande importance, mais toujours pour améliorer les processus et les services.

La question se pose de savoir pourquoi il n'existe pas de programme de recherche pour développer 
des nouvelles technologies par ceux qui sont habitués à les utiliser depuis 100 ans, afin d'arriver à une vrai souveraineté pétrolière. En fait, la réponse est évidente au regard de l'histoire du Venezuela : ni dans le complexe technique du café, ni dans celui du pétrole, le Vénézuela n'a été à l'origine des inventions techniques de production. La formation a toujours été limitée à l'utilisation des outils : l'invention est donc peu valorisée dans l'imaginaire technique vénézuelien.

Pourtant, avec une filière technologique ancienne de plus d'un siècle, il serait raisonnable de penser à son amélioration. Alors que l'industrie pétrolière vénézuélienne est dans un processus d'acclimatation, il est nécessaire, pour passer à l'étape suivante de la naturalisation, de retravailler le lien entre l'université et l'industrie, aussi bien dans des dimensions historiques (comment s'est constitué cette filière et ce complexe technique) que prospectives.

Dans ce cadre, l'analyse de l'histoire des techniques permettra d'envisager l'industrie pétrolière comme étant un patrimoine industriel et non plus uniquement comme des structures de nature uniquement économique. Il permettra aussi de voir l'avantage que représente le fait qu'il soit encore en activité au Venezuela, par rapport à l'expérience des autres pays, notamment en termes de prédictibilité. Conserver la mémoire historique, à travers son patrimoine, c'est alimenter les signaux d'identité d'un pays qui ne veut pas voir annulé son rôle dans le monde. Il est donc nécessaire de poser comme point de départ la recherche historique, suivi de l'inventaire du patrimoine industriel pétrolier au niveau national, pour «définir clairement les objectifs de base de l'intervention, les plus importants étant l'intégration de ce patrimoine dans un territoire en respectant strictement ses caractéristiques, sa préservation, l'éducation à ce patrimoine, et sa dissémination, entre autres ${ }^{10}$. Il s'agit ici d'orienter nos efforts en plaçant le paysage non comme la conséquence de l'action d'une culture, mais comme notre réalité, notre identité et notre mémoire dans une évolution continue.

\footnotetext{
${ }^{1}$ Nous utilisons la notion telle qu'elle est développée par Bertrand Gille et complétée par Anne-Françoise Garçon, qui remet en question le caractère globalisant de la notion dans le temps et l'espace: moins que la stabilité et l'universalité du système, c'est l'interdépendance de ces composantes qui est donc mis en avant.

2 Rappelons que la Standard Oil de Rockfeller fut fondé en 1870.

${ }^{3}$ SULLIVAN W., "Situación económica y política durante el período de Juan Vicente Gómez", Política y Economía en Venezuela 1810-1991, Caracas, Fundación Boulton, 1992: p. 258.

${ }^{4}$ CILENTO A. Cambio de paradigma del habitat. Caracas, IDECCDCH/UCV, Colección Estudios, 1999, p. 140.

${ }^{5}$ HOWARD E., Garden Cities of Tomorrow, Swan Sonneschein, 1902, p. 15 .

6 GONZÁLEZ L. et MARÍN O. "El transcurrir tras el cercado: ámbito residencial y vida cotidiana en los campamentos petroleros de Venezuela (1940-1975)", Espacio Abierto, Universidad del Zulia, 2003, p.6.

${ }^{7}$ TAYLOR W., et LINDEMAN J., Vénézuéla sows its petroleum, National Planning Association, 1955, p. 14.

8 Comité International pour la sauvegarde du patrimoine Industriel.

9 Plan implémenté par PDVSA depuis 2005 pour l'investissement de la remise pétrolière dans les actions de caractère social.

1o SABATÉ J., "De la Preservación del Patrimonio a la Ordenación del Paisaje”, Urbano, Universidad del Biobío, 2004, p. 49.
} 


\section{$\underline{\text { Bibliographie }}$}

ARNOLD R., MACREADY G. A. et BARRINGTON T. W., The First Big Oil Hunt, Venezuela 1911-1916, New-York, Vantage Press Inc., 1960.

\section{BALESTRINI C. Economía Minera $y$} Petrolera, Caracas, Universidad Central de Venezuela, 1959.

CILENTO A. Cambio de paradigma del habitat. Caracas, IDEC-CDCH/UCV, Colección Estudios, 1999.

GARÇON A., L'imaginaire et la pensée technique - Une approche historique, XVIe-XXe siècle, Paris, Classiques Garnier, 2012.

GARÇON A., Techniques et innovation : acteurs, idées, territoires, XVIIIe- XXe siècles. HAL-SHS, 2006.

GILLE B., Morphologie des techniques - École pratique des hautes études. $4^{e}$ section, Sciences historiques et philologiques. Annuaire 1972-1973: p. 537 .

GILLE B., " La notion de " système technique » (essai d'épistémologie technique) », Technique et culture, $\mathrm{n}^{\circ} 1$, octobre 1979 , p. 8 - 18 .

GONZÁLEZ L. et MARÍN O. "El transcurrir tras el cercado: ámbito residencial y vida cotidiana en los campamentos petroleros de Venezuela (19401975)", Espacio Abierto, Universidad del Zulia, 2003.

HOWARD E., Garden Cities of Tomorrow, Swan Sonneschein, 1902.

MARTÍNEZ A., Cronología del Petróleo en Venezuela, Purnell and Sons LTD, 1969.

SABATÉ J., "De la Preservación del Patrimonio a la Ordenación del Paisaje", Urbano, Universidad del Biobío, 2004, p. 49.
SULLIVAN W., "Situación económica y política durante el período de Juan Vicente Gómez", Política y Economía en Venezuela 1810-1991, Caracas, Fundación Boulton, 1992: p. 258.

TAYLOR W., et LINDEMAN J., Vénézuéla sows its petroleum, National Planning Association, 1955.

VALERY R., Las Comunidades Petroleras, Caracas, Cuadernos Lagoven, 1980. 